\title{
Effect of coupling currents on the dynamic inductance during fast transient in superconducting magnets
}

\author{
V. Marinozzi, ${ }^{1}$ M. Sorbi, ${ }^{1}$ G. Manfreda, ${ }^{2}$ F. Bellina, ${ }^{2}$ H. Bajas, ${ }^{3}$ and G. Chlachidze ${ }^{4}$ \\ ${ }^{1}$ University of Milan and INFN-LASA, 20090 Milano, Italy \\ ${ }^{2}$ University of Udine, 33100 Udine, Italy \\ ${ }^{3}$ CERN, TE department, 1211 Geneva, Switzerland \\ ${ }^{4}$ Fermilab-TD department, Batavia, Illinois 60510-5011, USA \\ (Received 17 December 2014; published 26 March 2015)
}

\begin{abstract}
We present electromagnetic models aiming to calculate the variation of the inductance in a magnet due to dynamic effects such as the variation of magnetization or the coupling with eddy currents. The models are studied with special regard to the calculation of the inductance in superconducting magnets which are affected by interfilament coupling currents. The developed models have been compared with experimental data coming from tests of prototype $\mathrm{Nb}_{3} \mathrm{Sn}$ magnets designed for the new generation of accelerators. This work is relevant for the quench protection study of superconducting magnets: quench is an unwanted event, when part of the magnet becomes resistive; in these cases, the current should be discharged as fast as possible, in order to maintain the resistive zone temperature under a safe limit. The magnet inductance is therefore a relevant term for the description of the current discharge, especially for the high-field new generation superconducting magnets for accelerators, and this work shows how to calculate the correct value during rapid current changes, providing a mean for simulations of the reached temperature.
\end{abstract}

DOI: 10.1103/PhysRevSTAB.18.032401

PACS numbers: $84.71 . \mathrm{Ba}$

\section{INTRODUCTION}

Superconducting magnets are a technology of paramount importance in particle accelerators because of the high performances that they can offer in terms of field gradient and quality [1]. However, magnetothermal instabilities (quenches) could affect their conductors, inducing a transition from a superconducting to a normal-conducting state. In these cases, a protection system must discharge the stored magnetic energy as fast as possible, in order to protect the windings from overheating. For the next generation of high-field superconducting magnets $\left(B_{\text {peak }} \approx 12-15 \mathrm{~T}\right)$, the use of $\mathrm{Nb}_{3} \mathrm{Sn}$ as a superconductor is foreseen. Because of the large ratio between the magnetic energy and the conductor mass, the quench protection is one of the most challenging aspects for the design of these magnets [2], and in the future it could be the limiting factor for them. The protection problem is more relevant in highfield dipole and quadrupole magnets for accelerators than in other high-field magnets, such as solenoids: in fact, the first ones require very large values of current density in the coils, whereas in the second ones, usually, stabilized conductors can be used, with much lower current density in the coil area.

Published by the American Physical Society under the terms of the Creative Commons Attribution 3.0 License. Further distribution of this work must maintain attribution to the author(s) and the published article's title, journal citation, and DOI.
Some experimental results [3] showed that in some superconducting magnets, during a fast current decay, the inductance appears lower than expected; in particular, in some cases, the inductance is 30\% lower [4] than the value measured at low $d I / d t$. The quench protection of magnets with high field and high energy density can sensibly benefit from this inductance reduction, because the current discharge during a quench results faster. As a consequence, a deep understanding and prediction of the phenomenon could play an important role for the design of a safe protection for such superconducting magnets [5].

Here we explain this inductance reduction considering the magnetic flux change due to the coupling currents in the wires. The presented model can be generalized to any energy loss due to magnetization phenomena (both magnetic materials and magnetization currents). The energy variations due to the magnetization are manifested as a change in the differential inductance.

We present two approaches for the calculation of the inductance variation due to a magnetization phenomenon in a magnet; then we present two ways for the calculation of the inductance in a superconducting magnet during rapid current changes, such as during a quench, when large interfilament coupling currents (IFCCs) are generated, showing how to compute the magnetization due to the IFCC; finally, we compare the results with experimental data, showing an analytical example of a long superconducting quadrupole magnet and a numerical example of a short racetrack magnet. 


\section{INDUCTANCE VARIATION DUE TO MAGNETIZATION}

During current changes, the voltage across a superconducting cable is given by

$$
V=-\frac{d \phi}{d t}=-\frac{d \phi}{d I_{t}} \frac{d I_{t}}{d t}
$$

where $I_{t}$ indicates the transport current. The "standard" inductance is defined as

$$
L_{s}=\frac{\phi}{I_{t}}
$$

therefore, Eq. (1) can be written as

$$
V=-\frac{d\left(L_{s} I_{t}\right)}{d I_{t}} \frac{d I_{t}}{d t}=-\left(L_{s}+\frac{d L_{s}}{d I} I_{t}\right) \frac{d I_{t}}{d t}
$$

We can now define the differential inductance

$$
L_{d}=L_{s}+I_{t} \frac{d L_{s}}{d I_{t}}=\frac{d \phi}{d I_{t}},
$$

which is equivalent to the definition (2) only when the inductance does not depend on the current. Since the usual inductance measurements as $V / \dot{I}$ give as the result the differential inductance, we will consider $L_{d}$, which will be called simply $L$ from now on.

The differential inductance is computed now by following two different approaches. The first one is based on an energetic model, the second one on a vector potential analysis.

\section{A. Energetic approach}

Suppose that, during a current variation, a magnetization phenomenon occurs in a superconductor, and a magnetization $M$ is produced; the total magnetic induction field on the superconductor is

$$
B=\mu_{0} M+\mu_{0} H .
$$

The magnetic susceptibility $\chi$ can be used in order to express the magnetization in terms of the magnetic field

$$
M=\chi H
$$

therefore, the magnetic energy increment per volume unit $d u=H d B$ can be expressed as

$$
d u=\mu_{0} H d H+\mu_{0} \chi H d H+\mu_{0} H^{2} d \chi .
$$

The magnetic energy variation per time unit is

$$
-\frac{d U}{d t}=V I_{t}
$$

Combining Eqs. (3), (4), and (8), we obtain

$$
\frac{d U}{d t}=L I_{t} \frac{d I_{t}}{d t}
$$

therefore, we can write the inductance as

$$
L=\frac{1}{I_{t}} \frac{d U}{d I_{t}} .
$$

Combining Eqs. (7) and (10), we obtain

$$
L=\int\left\{\frac{\mu_{0} H}{I_{t}} \frac{d H}{d I_{t}}+\frac{\mu_{0} \chi H}{I_{t}} \frac{d H}{d I_{t}}+\frac{\mu_{0} H^{2}}{I_{t}} \frac{d \chi}{d I_{t}}\right\} d V .
$$

Equation (11) expresses the inductance calculated by considering the occurrence of magnetization phenomena. Generally, the magnetization involves a change not only of $B$, through $M$, but also of $H$, due to the presence of a demagnetization field $H_{d m}$; therefore, all three terms in (11) contribute to the differential inductance variation. However, in some cases, such as during a fast discharge in a superconducting magnet, the demagnetization field is small compared to the $H_{0}$ field generated by the transport current in air. As a consequence, the variation of inductance is due to the second and mainly to the third term in (11), while the first term can be considered representative of the static inductance. The problem of magnetization during a quench in superconducting magnets will be discussed in Sec. III.

\section{B. Vector potential approach}

The magnetic vector potential that arises in a point $P(\boldsymbol{r})$ from a generic distribution of current and magnetized media located in a volume $V$ can be computed as

$\boldsymbol{A}(\boldsymbol{r})=\frac{\mu_{0}}{4 \pi}\left[\int_{V}\left(\frac{\boldsymbol{J}_{t}}{\left|\boldsymbol{r}-\boldsymbol{r}^{\prime}\right|}+\frac{\boldsymbol{J}_{m v}}{\left|\boldsymbol{r}-\boldsymbol{r}^{\prime}\right|}\right) d^{3} \boldsymbol{r}^{\prime}+\int_{S} \frac{\boldsymbol{J}_{m s}}{\left|\boldsymbol{r}-\boldsymbol{r}^{\prime}\right|} d^{2} \boldsymbol{r}^{\prime}\right]$,

where $\boldsymbol{J}_{t}$ is the transport current density, $S$ is the surface of the magnetized media (with outwards normal $\boldsymbol{n}$ ), and

$$
\begin{aligned}
\boldsymbol{J}_{m v} & =\boldsymbol{\nabla} \times \boldsymbol{M}, \\
\boldsymbol{J}_{m s} & =\mathbf{M} \times \boldsymbol{n}
\end{aligned}
$$

are the equivalent volume and boundary surface current densities, respectively, that represent the magnetized material. An approach based on this magnetic vector potential $\boldsymbol{A}$ for the computation of the effect of magnetization on magnet inductance is therefore also possible. 
Since $\boldsymbol{M}=\chi \boldsymbol{H}$, we can rewrite the volume equivalent current density $\boldsymbol{J}_{m v}$ as

$$
\boldsymbol{J}_{m v}=\chi \boldsymbol{\nabla} \times \boldsymbol{H}+\boldsymbol{\nabla} \chi \times \boldsymbol{H} .
$$

However, considering a uniform susceptibility $(\nabla \chi=0)$ and combining $\boldsymbol{\nabla} \times \boldsymbol{H}=\boldsymbol{J}_{t}$, Eq. (12) becomes

$$
\begin{aligned}
\boldsymbol{A}(\boldsymbol{r}) & =(1+\chi) \frac{\mu_{0}}{4 \pi} \int_{V} \frac{\boldsymbol{J}_{t}}{\left|\boldsymbol{r}-\boldsymbol{r}^{\prime}\right|} d^{3} \boldsymbol{r}^{\prime}+\frac{\mu_{0}}{4 \pi} \int_{S} \frac{\boldsymbol{J}_{m s}}{\left|\boldsymbol{r}-\boldsymbol{r}^{\prime}\right|} d^{2} \boldsymbol{r}^{\prime} \\
& =(1+\chi) \boldsymbol{A}_{t}(\boldsymbol{r})+\boldsymbol{A}_{m s}(\boldsymbol{r}),
\end{aligned}
$$

in which $\boldsymbol{A}_{t}$ is the transport current $\boldsymbol{J}_{t}$ contribution to the magnetic vector potential, whereas $\boldsymbol{A}_{m s}$ is due to the distribution of current per unit length $\boldsymbol{J}_{m s}$. The total magnetic flux $\phi$ can be therefore written as

$$
\phi=(1+\chi) \phi_{t}+\phi_{m s},
$$

where $\phi_{t}$ and $\phi_{m s}$ are, respectively, the circulations of $\boldsymbol{A}_{t}$ and $\boldsymbol{A}_{m s}$ along the path $\Gamma$ that edges the surface of interest $\left(\phi=\oint_{\Gamma} \boldsymbol{A} \cdot \boldsymbol{d} \boldsymbol{l}\right)$. Expressing the current densities $\boldsymbol{J}_{t}$ and $\boldsymbol{J}_{m s}$, respectively, as suitable time-independent functions multiplied for the time-dependent currents $I_{t}$ and $I_{m s}$, the fluxes can be written as

$$
\begin{gathered}
\phi_{t}=\phi_{t, 1} I_{t}, \\
\phi_{m s}=\phi_{m s, 1} I_{m s},
\end{gathered}
$$

where $\phi_{t, 1}$ and $\phi_{m s, 1}$ are the magnetic fluxes computed, respectively, for a unitary transport current $I_{t}$ and a unitary surface magnetization current $I_{m s}$. The differential inductance can therefore be written by using Eq. (4)

$$
L=\left[\left(1+\chi+\frac{d \chi}{d I_{t}}\right) \phi_{t, 1}+\frac{d I_{m s}}{d I_{t}} \phi_{m s, 1}\right],
$$

where $d \chi / d I_{t}$ and $d I_{m s} / d I_{t}$ are, respectively, the derivatives of the magnetic susceptibility and of the surface magnetization current with the transport current. In the absence of magnetization $\left(\chi, I_{m s}=0\right)$, the inductance is

$$
L=\phi_{t, 1},
$$

which is equivalent to the "standard" inductance.

\section{EFFECT OF IFCC ON THE INDUCTANCE}

During a quench in a superconducting magnet, the current derivative reaches very high values, from 1 to $100 \mathrm{kA} / \mathrm{s}$. In these conditions, a considerable susceptibility variation arises, due to the large coupling currents that are generated in the wires. In this section, we show how to calculate the magnetization and the related susceptibility due to IFCC in two different cases, which have wide applicability: when the applied magnetic field can be approximated with an exponential function of time or with a power series.

\section{A. General model for IFCC}

Superconducting wires are composed by a large number of filaments embedded in a normal-conducting (usually copper) matrix. When they are exposed to time-varying fields, coupling currents can flow among filaments through the normal conducting matrix. It can be proved [6] that the magnetic field $B_{i}$ acting on the superconducting wires, when IFCCs are present, follows the equation

$$
B_{i}=B_{e}-\frac{d B_{i}}{d t} \tau
$$

where $B_{e}$ is the applied field and $\tau$ is the IFCC decay time constant, which is [6]

$$
\tau=\frac{\mu_{0}}{2 \varrho_{e}}\left(\frac{p}{2 \pi}\right)^{2}
$$

where $p$ is the filament twist pitch in the wire and $\varrho_{e}$ is the effective transverse resistivity of the copper matrix. In this study, we assume that $\tau$ is constant; therefore, we neglect the copper magnetoresistance. Typical values for $\tau$ are 1-100 ms, taking into account the uncertainties on the matrix transverse resistivity [7] and the typical values of the twist pitch [8].

By means of the internal field $B_{i}$, it is possible to calculate the magnetization due to the IFCCs from direct integration of the coupling currents distribution (for more details, see [6])

$$
M=\frac{2 \lambda \tau}{\mu_{0}} \frac{d B_{i}}{d t},
$$

where $\lambda$ takes into account the presence of the insulation in the cable (that is not magnetized) and the conductor packing factor (filaments do not fill uniformly the strand); typical values are $\lambda \simeq 0.7-0.8$. By combining Eqs. (5), (6), and (24), it is possible to express the susceptibility related to the IFCCs:

$$
\chi=-\frac{1}{1+\frac{1}{2 \lambda}\left(\frac{1}{1-B_{e} / B_{i}}\right)} .
$$

From Eq. (25), note that, under the assumption of constant $\tau$, the susceptibility is uniform along the magnet, since it depends only on the ratio $B_{e} / B_{i}$; therefore, the wires can be considered as a uniform magnetized material. 


\section{B. Exponential approach}

Assuming that $B_{e}$ decreases exponentially

$$
B_{e}=B_{0} e^{-\left(t / \tau_{e}\right)},
$$

together with the initial condition

$$
B_{i}(0)=B_{e}(0)=B_{0},
$$

the solution of Eq. (22) can be found as

$$
B_{i}=\frac{B_{0}}{\tau-\tau_{e}}\left(\tau e^{-(t / \tau)}-\tau_{e} e^{-\left(t / \tau_{e}\right)}\right) .
$$

By combining Eqs. (25), (26), and (28), the magnetic susceptibility can be obtained:

$$
\chi=\frac{2 \lambda \tau\left(e^{-\left(t / \tau_{e}\right)}-e^{-(t / \tau)}\right)}{\tau e^{-(t / \tau)}-\tau_{e} e^{-\left(t / \tau_{e}\right)}-2 \lambda \tau\left(e^{-\left(t / \tau_{e}\right)}-e^{-(t / \tau)}\right)} .
$$

The inductance at this point can be calculated by using Eq. (11) or (20).

\section{Power series approach}

Another possibility is to express the time dependence of the applied field as a power series:

$$
B_{e}=\sum_{i=0}^{N} \alpha_{i} t^{i}
$$

This is particularly useful in the case of experimental data that can be fitted with a polynomial or a spline. Obviously, the case of the exponential can be reduced to this case considering a sufficiently large value for $N$ and a proper choice of $\alpha_{i}$, i.e., the Taylor series coefficients.

Equation (22) can be rewritten in the classical shape of the ordinary differential equations:

$$
\frac{d B_{i}}{d t}+\frac{B_{i}}{\tau}=\frac{B_{e}}{\tau},
$$

whose solution is

$$
B_{i}=B_{0} e^{-(t / \tau)}+\frac{e^{-(t / \tau)}}{\tau} \int_{0}^{t} B_{e}(s) e^{s / \tau} d s
$$

Inserting (30) in (32) gives

$$
B_{i}=B_{0} e^{-(t / \tau)}+\frac{e^{-(t / \tau)}}{\tau}\left(\sum_{i=0}^{N} \alpha_{i} \int_{0}^{t} s^{i} e^{s / \tau} d s\right) .
$$

With the change of variables $x=s / \tau$, it becomes

$$
B_{i}=B_{0} e^{-(t / \tau)}+e^{-(t / \tau)}\left(\sum_{i=0}^{N} \alpha_{i} \tau^{i} \int_{0}^{t / \tau} x^{i} e^{x} d x\right) .
$$

The general solution for the integral in Eq. (34) is [9]

$$
\int x^{i} e^{x} d x=(-1)^{i} i ! e^{x} \sum_{j=0}^{i} \frac{(-x)^{j}}{j !} .
$$

By combining (34) and (35), the result is

$$
B_{i}=B_{0} e^{-(t / \tau)}+\sum_{i=0}^{N} i ! \alpha_{i}(-\tau)^{i}\left[1-e^{-(t / \tau)}+\sum_{j=1}^{i} \frac{1}{j !}\left(-\frac{t}{\tau}\right)^{j}\right] .
$$

Imposing the initial condition, that is, for a nonmagnetized material

$$
B_{i}(0)=B_{e}(0)=\alpha_{0},
$$

the final result becomes

$$
B_{i}=\alpha_{0}+\sum_{i=0}^{N} i ! \alpha_{i}(-\tau)^{i}\left[1-e^{-(t / \tau)}+\sum_{j=1}^{i} \frac{1}{j !}\left(-\frac{t}{\tau}\right)^{j}\right] .
$$

The magnetization and the susceptibility can be found as just done for Eqs. (24) and (29), and then the inductance can be calculated by using Eq. (11) or (20).

\section{ANALYTICAL CALCULATION OF THE INDUCTANCE REDUCTION IN A LONG MAGNETIC QUADRUPOLE}

A long magnetic quadrupole is a good example for an analytical calculation of the inductance reduction. Indeed, the magnetic field in such a magnet can be easily expressed through analytical formulas, and this allows one to obtain simple relations using the energetic approach shown above, without the need of computing the vector potential.

\section{A. Inductance calculation}

In Eq. (11), assuming that $H$ is not affected by the magnetization effects and considering that $H d H=d H^{2} / 2$, the inductance variation is

$$
\Delta L=\frac{\mu_{0} \chi}{2 I_{t}} \frac{d}{d I_{t}} \int H^{2} d V+\frac{\mu_{0}}{I_{t}} \frac{d \chi}{d I_{t}} \int H^{2} d V .
$$

The presence of the two terms $\chi$ and $d \chi / d I$ allows one to limit the integration domain to the coil volume, because these two terms are null elsewhere. For simple geometries, the magnetic field can be obtained and integrated 
analytically; otherwise, the field map on the coils can be obtained and integrated by using numerical methods.

A 2D geometry is considered in which a $\cos 2 \vartheta$ magnetic field is assumed. This is accurate for long accelerator quadrupole magnets that are characterized by high field quality; otherwise, a 3D numerical model should be developed.

The magnetic field $B_{0}$ produced inside the coils by a volumetric transport current distribution $J=J_{0} \cos 2 \vartheta$ can be expressed by using the complex formalism [10]

$$
B_{0}^{*}=i \frac{\mu_{0} J_{0}}{2}\left[z \ln \left(\frac{a_{2}}{|z|}\right)-\frac{1}{4} \frac{|z|^{4}-a_{1}^{4}}{z^{3}}\right],
$$

where $a_{2}$ and $a_{1}$ are, respectively, the outer and the inner radius of the coils, $z=x+i y$ is the coordinate inside the coils $\left(a_{1} \leq|z| \leq a_{2}\right)$, and $B^{*}=B_{x}-i B_{y}$ ( $i$ is the pure imaginary unit). $H$ can be obtained from the relation

$$
H \simeq \frac{B_{0}}{\mu_{0}}
$$

neglecting the demagnetization field $H_{d m}$, which is assumed to be small compared to the field due to the transport current; this approximation is true for typical values of the magnetization $M$ [Eq. (27)]. By using the relation $H^{2}=H^{*} H$ and using the cylindrical coordinates $z=r e^{i \vartheta}$, the magnetic field intensity can be obtained:

$H^{2}=\frac{J_{0}^{2}}{4}\left[r^{2} \ln ^{2} \frac{a_{2}}{r}+\frac{1}{16} \frac{\left(r^{4}-a_{1}^{4}\right)^{2}}{r^{6}}-\frac{1}{2} \frac{r^{4}-a_{1}^{4}}{r^{2}} \ln \frac{a_{2}}{r} \cos 4 \vartheta\right]$.

The result of the volume integration on the coil volume is

$$
\begin{aligned}
& l \int_{a_{1}}^{a_{2}} \int_{0}^{2 \pi} H^{2} r d r d \vartheta \\
& \quad=\frac{l J_{0}^{2} \pi}{2}\left\{\frac{3}{64} a_{2}^{4}-\frac{a_{1}^{4}}{64}\left[16\left(\ln ^{2} \frac{a_{2}}{a_{1}}+\ln \frac{a_{2}}{a_{1}}\right)+\frac{a_{1}^{4}}{a_{2}^{4}}+2\right]\right\},
\end{aligned}
$$

where $l$ is the magnet length.

The current derivative in the first term of Eq. (39) can be calculated by noting that

$$
N I_{t}=2 \int_{a_{1}}^{a_{2}} \int_{-(\pi / 4)}^{\pi / 4} J_{0} \cos 2 \vartheta r d r d \vartheta=J_{0}\left(a_{2}^{2}-a_{1}^{2}\right),
$$

where $N$ is the total number of turns, so we obtain

$$
\frac{d H^{2}}{d I_{t}}=\frac{d H^{2}}{d J_{0}} \frac{N}{\left(a_{2}^{2}-a_{1}^{2}\right)}=\frac{2 N H^{2}}{J_{0}\left(a_{2}^{2}-a_{1}^{2}\right)} .
$$

The inductance reduction caused by the IFCC can therefore be found by replacing the results (29), (43), and (45) in Eq. (39).

\section{B. Implementation in QLASA}

QLASA is a computer code for the quench simulation [11]. The program simulates a standard quench protection circuit such as the one shown in Fig. 1.

The magnet is represented by its inductance $L$, and $R_{q}$ is its resistance developed during the quench propagation (therefore, it is time dependent). When the quench is detected, the switch $S 1$ is opened, and the current is forced to flow into the dump resistor $R_{d}$, which extracts part of the energy stored into the magnet.

The current decay is so computed by solving with a temporal step-by-step method the equation

$$
\left(R_{d}+R_{q}\right) I_{t}+L \frac{d I_{t}}{d t}=0
$$

Details on the program operation can be found in [11]. QLASA is therefore suitable for implementing the method described in Sec. III B: at each time step the inductance is recalculated according to Eq. (39), and the current decreases according to Eq. (46); the resistance and the inductance are assumed to be constant, and therefore the output current from (46) is a piecewise exponential. This justifies the assumption (24), provided that the decay time $\tau_{e}=L /\left(R_{d}+R_{q}\right)$ is recalculated at each time step. The program calculates the resistance growth due to the quench, combining quench propagation and heat produced by the Joule effect. If IFCCs are present, anyway, there is also an additional power dissipation, which can be calculated through the magnetization: the power per volume unit dissipated by the IFCCs is [6]

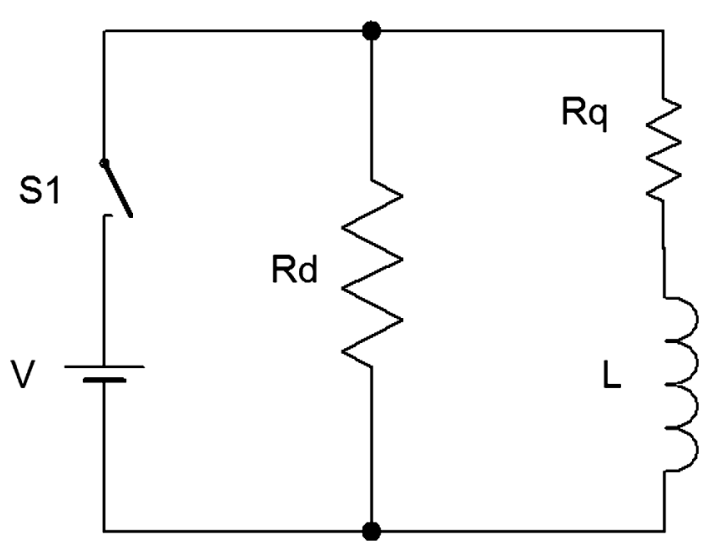

FIG. 1. Schematic quench protection circuit. 


$$
P_{\mathrm{IFCC}}=\frac{2 \lambda \tau}{\mu_{0}}\left(\frac{d B_{i}}{d t}\right)^{2} .
$$

Equation (47), combined with Eq. (26), becomes

$$
P_{\mathrm{IFCC}}=\frac{2 \lambda \tau}{\mu_{0}}\left[\frac{B_{0}}{\tau-\tau_{e}}\left(e^{-\left(t / \tau_{e}\right)}-e^{-(t / \tau)}\right)\right]^{2} .
$$

This expression has been implemented in the QLASA subroutine which computes the magnet temperature and the quench resistance. More details on the resistance computation can be found in Ref. [11].

\section{Experimental comparison}

The model implemented in QLASA has been compared with some experimental data from quench tests of R\&D magnets for the project High Luminosity LHC (HiLumi) [5].

Here we present a test done on HQ [12], a $1 \mathrm{~m}$ long $\mathrm{Nb}_{3} \mathrm{Sn}$ superconducting quadrupole, with a bore diameter of $120 \mathrm{~mm}$. The magnet nominal inductance ranges from $\sim 5.7 \mathrm{mH}$ at high current to $\sim 6.4 \mathrm{mH}$ at low current, being the difference due to the iron saturation, and it has been experimentally measured at low $d I / d t$ [13]. The test has been performed by extracting the magnet energy on an external dump resistor with a resistance of $60 \mathrm{~m} \Omega$. In this measurement there is no natural quench, and the starting current is $13 \mathrm{kA}$, which is the $80 \%$ of the short sample limit [12]. By neglecting the iron saturation, which is important only at the end of the discharge, the current decay should be a simple exponential, with a time constant $\bar{\tau}=L / R_{d} \simeq 0$, $1 \mathrm{~s}$. However, comparing the experimental decay with the exponential curve $I(t)=I_{0} e^{-(t / \bar{\tau})}$, there is not agreement between the two curves, as shown in Fig. 2.

The experimental decay is appreciably faster than expected; in fact, the actual decay time constant is $30 \%$ lower than the expected one. This happens at the very beginning of the decay, when such a difference cannot be

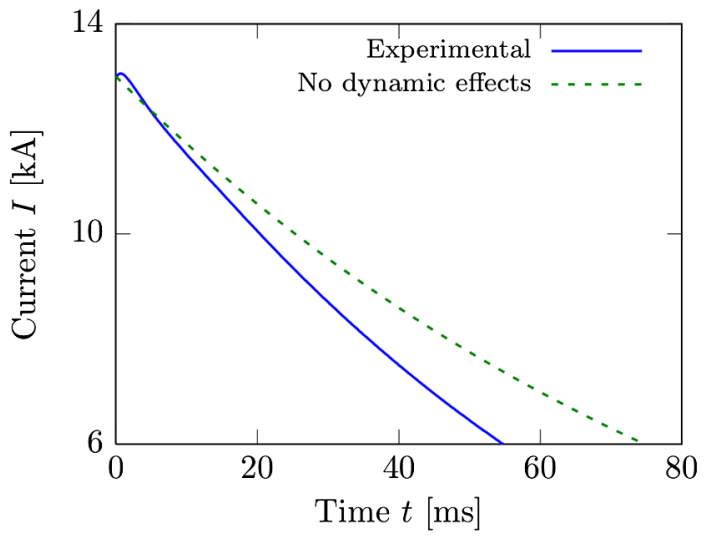

FIG. 2. Comparison between the experimental current decay (solid blue curve) and a pure exponential (dashed green curve).

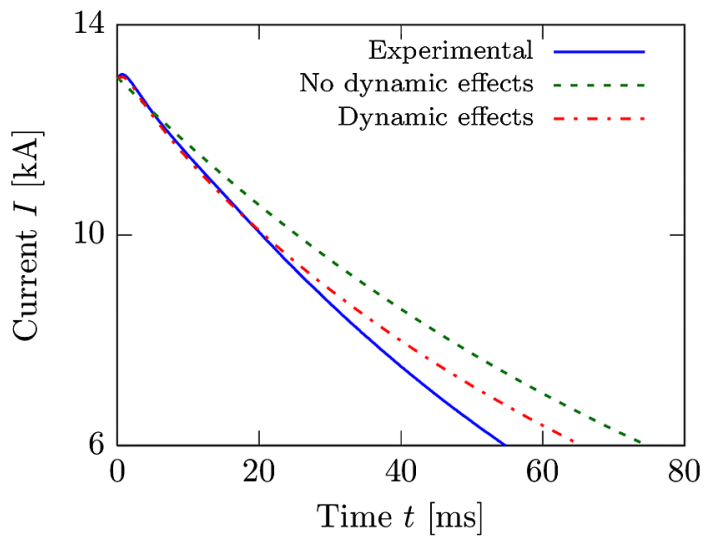

FIG. 3. Comparison between the experimental current decay (solid blue curve), a pure exponential (dashed green curve), and a QLASA simulation which considers dynamic effects on the inductance due to IFCCs (point-dashed red curve).

justified by a quench back. Quench back [6] is a phenomenon strictly related to IFCC: the coupling currents, flowing through the copper matrix, dissipate some heat inside the wire, raising its temperature and inducing a quench; this phenomenon, anyway, usually needs at least $\sim 5-10 \mathrm{~ms}$ to occur.

The test has been modeled with QLASA, considering the dynamic IFCC effects on the magnet inductance, as described in Secs. IVA and IV B. The result is shown in Fig. 3. The agreement is good at the beginning of the decay, until $\sim 10 \mathrm{~ms}$. Later on, the experimental current decay becomes too fast. In order to explain this feature, we assumed the occurrence of a quench back after $10 \mathrm{~ms}$. QLASA presently cannot detect the occurrence of a quench back by itself but can model it once the appropriate initial

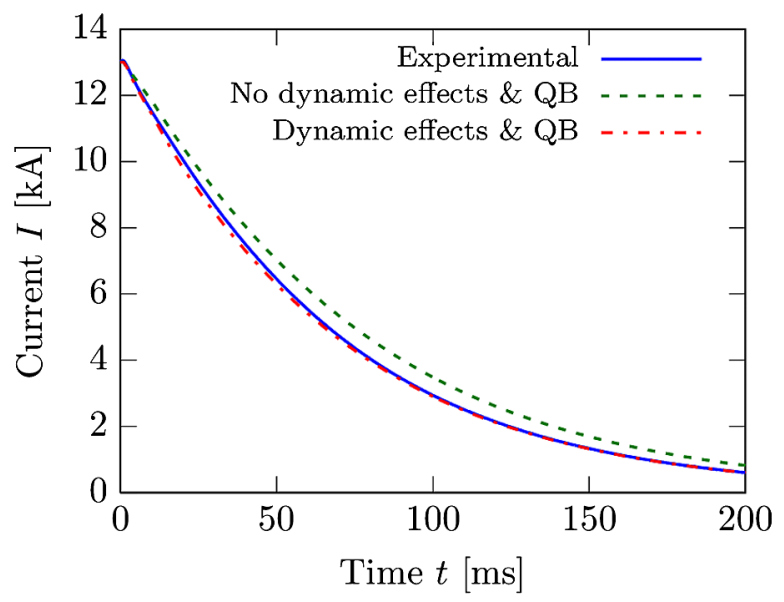

FIG. 4. Comparison between the experimental current decay (solid blue curve), a QLASA simulation which considers dynamic effects on the inductance due to IFCCs and quench back after $10 \mathrm{~ms}$ (point-dashed red curve), and a standard QLASA simulation without considering dynamic effects but considering quench back after $10 \mathrm{~ms}$ (dashed green curve). 
conditions are set. In this way, the starting time of a quench back in QLASA can be viewed as a sort of fitting parameter, which has been set to $10 \mathrm{~ms}$ in the present case. In Fig. 4, it can be noted that the simulation made considering dynamic IFCC effects and quench back fits well the experimental curve. Comparing Figs. 3 and 4, it is easy to notice that quench back plays a very important role in the decay. On the other hand, quench back alone cannot justify the experimental behavior, as can be seen in Fig. 4 (dashed green curve).

The conclusion is that the dynamic effects due to IFCC and the related quench back are both needed for a good simulation of a fast current decay. To improve the simulation accuracy, the implementation of the code in order to predict the occurring of quench back is in progress.

\section{NUMERICAL COMPUTATION OF THE INDUCTANCE REDUCTION IN A SMALL RACETRACK MAGNET}

When the field produced by the magnet cannot be expressed by an analytical formula like Eq. (40), the previous method is no longer applicable. An example of this is the short model coil, a small $\mathrm{Nb}_{3} \mathrm{Sn}$ racetrack magnet produced and tested by CERN [14], which has reached a peak field of about $13.5 \mathrm{~T}$ with a transport current of about $15.9 \mathrm{kA}$. The magnet winding is composed by two layers of 35 turns each, with a straight section about $15 \mathrm{~cm}$ long. A numerical approach for the $3 \mathrm{D}$ field computation is needed; therefore, the method shown above based on the potential vector has been applied.

In the present case, the contribution of the iron yoke to the electromagnetic field can be considered as a static background field of about 2 T. Indeed, we are interested in the inductive phenomena at very high fields, and in these conditions the iron is fully saturated. This is confirmed by the inductance measurement on the coil at low $d I / d t$ shown in Fig. 5, where a constant value of about $1.43 \mathrm{mH}$ for the

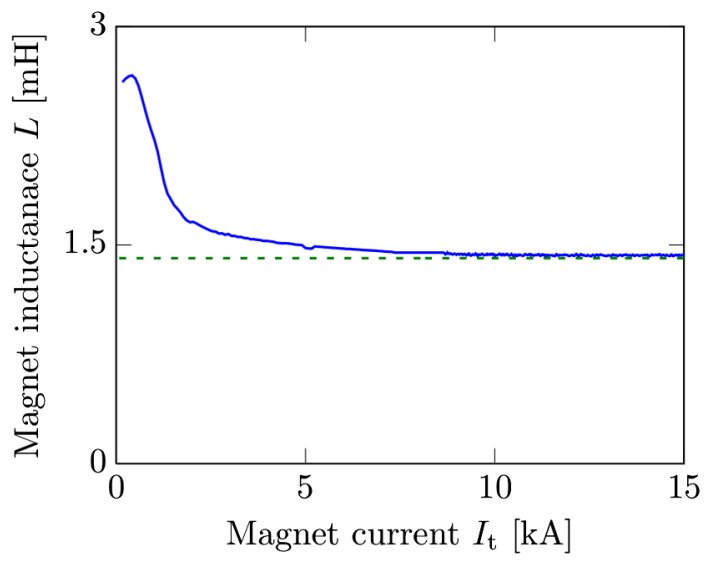

FIG. 5. Short model coil inductance as a function of the magnet current, measured at low $d I / d t$. The dashed line represents the computed value. inductance is obtained when the magnet transport current is over $10 \mathrm{kA}$, whereas variations are observed at lower current. We will therefore focus on the behavior of the magnet in fully saturated iron conditions.

\section{A. Transport current contribute}

The magnet geometry has been modeled with the program THELMA [15].

Each coil layer is modeled by using three solid racetracks with rectangular cross section, representing each group of turns divided by spacers. Each racetrack axis is modeled through two suitable rectilinear segments interleaved by two half-circular axes. In Fig. 6, a pictorial view of these solid racetracks is reported.

Taking advantage of the magnet symmetry, a threedimensional field map has been calculated in a grid of points in the positive octant $(x, y, z \geq 0)$ and then suitably extended by symmetry to the complete space needed for the computation. The distance between the points is about $1 \mathrm{~mm}$, and the field is linearly interpolated at the intermediate points. The circulation of $\boldsymbol{A}_{t}$ along all the magnet turns is then computed with the GNU Octave tool [16] using an adaptive Gauss-Konrod quadrature [17].

The resulting computed inductance of $1.41 \mathrm{mH}$ is in very good agreement with experimental data at high field, as shown by the dashed line in Fig. 5. This value corresponds to the contribution $\phi_{t, 1}$ of Eq. (20).

\section{B. Magnetization and susceptibility computation}

The considered magnet current discharge with time is reported in Fig. 7 together with its time derivative. The current starts to decay at about $10 \mathrm{~ms}$, when a natural quench happens in the conductor. The protection system features a dump resistor of $40 \mathrm{~m} \Omega$, which becomes conductive at about $2.8 \mathrm{~ms}$, assuming $t=0 \mathrm{~ms}$ as the moment when the quench is validated by the protection system. Very large values for the current derivative can be estimated, up to $40 \mathrm{kA} / \mathrm{s}$ before the switch opens and up to $900 \mathrm{kA} / \mathrm{s}$ after. As we see in Fig. 8, also in this case the exponential with a time constant $L / R_{d}$ is not able to reproduce the experimental data, if the static value of inductance is assumed. Moreover, the additional resistance developed on the coil by the quench cannot explain such a large discrepancy, with a time constant varying up to the 50\%. Equation (20) has been therefore employed to compute the dynamic inductance.

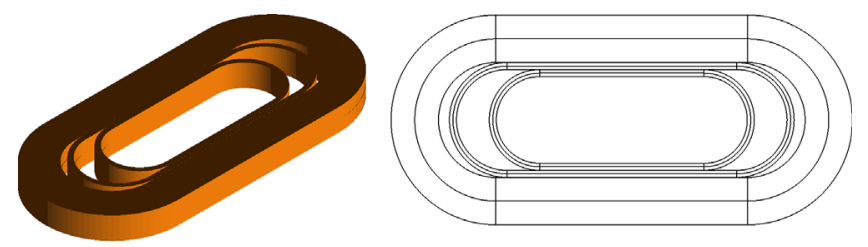

FIG. 6. Views of the modeled racetracks. 


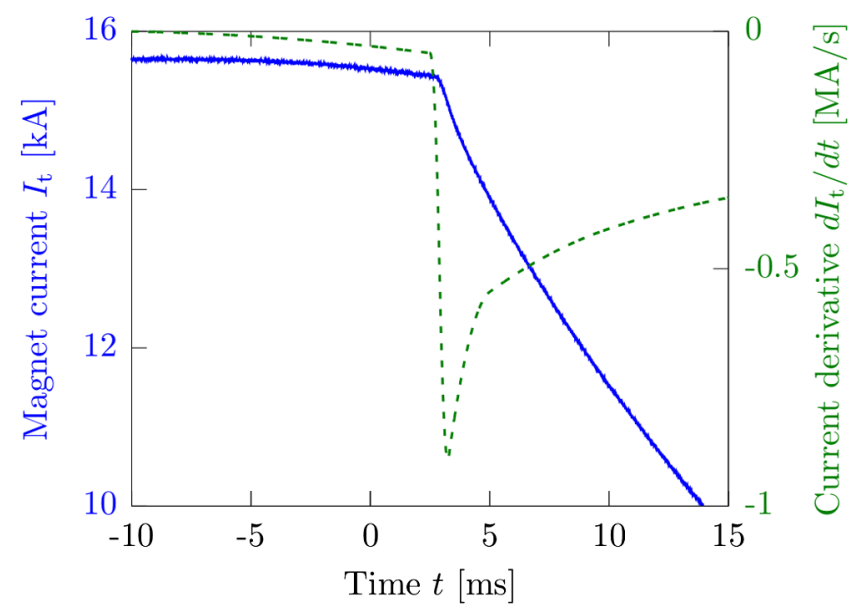

FIG. 7. Short model coil, magnet current, and its estimated derivative as a function of time after a quench starts.

The magnetic induction $B_{e}$ has been estimated as a function of time from the transport current by means of the magnet load line. The piecewise power series approach [Eq. (30)] has been employed, since data can be easily fitted with a cubic spline. Equation (36) has been used to compute $B_{i}$, whereas the magnetization $M$ (and therefore $I_{m s}$ ) and the susceptibility $\chi$ as a function of time are calculated through Eqs. (24) and (25).

As confirmed by the previous field computation, the magnetic induction in this type of racetrack magnet is by far dominated by the $z$ (out-of-plane) component, and the same applies also to the magnetization according to Eq. (24). Thus, in order to simplify the model and to reduce the computational effort required, we decided to consider only the $z$ component of the magnetization in the numerical model. This approximation leads to an underestimation of the effect only in the middle turns of the magnet, since in this region the $y$ (planar) component of the field can be significant. The computation of $\phi_{m s, 1}$ has been performed also in this case with the THELMA code. The vector

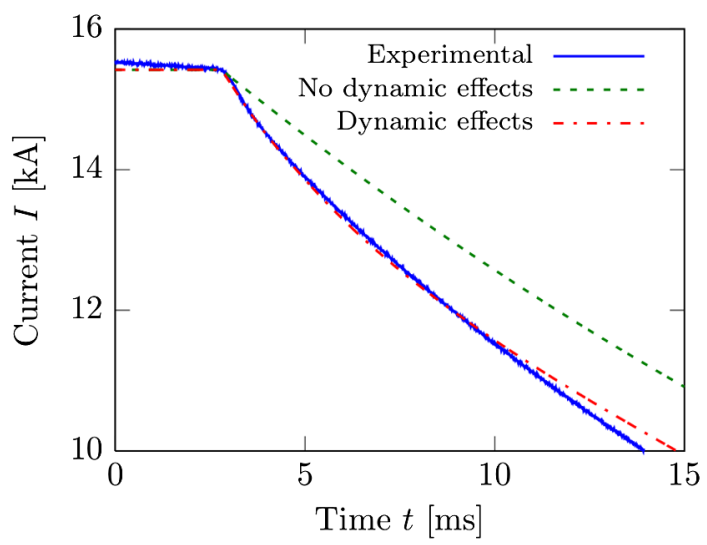

FIG. 8. Short model coil and magnet current decay compared with computed decays without and with dynamic effects. potential generated by $\boldsymbol{J}_{m s}$ has been computed by means of thin sheets of current placed in the inner, outer, and spacer surfaces of the coil parallel to the $z$ axis. The $\boldsymbol{J}_{m s}$ value has been suitably averaged along the turn where the sources are located. The resulting vector potential has been integrated with the same procedure explained in the previous section.

As we can see from Fig. 8, also in this case a very good fit on the data can be obtained including the dynamic effects in the inductance computation. The IFCC time constant has been set to $\tau=8 \mathrm{~ms}$ in this case, well within the expected range for this parameter. It is important to highlight that the method employed is applicable in the first $10-15 \mathrm{~ms}$ of the discharge. Indeed, when the current decays at lower values, the iron can no longer be considered saturated. Furthermore, as the coil becomes completely normal conducting, the IFCCs and their related magnetization vanish; therefore, the dynamic effects should vanish as well.

\section{CONCLUSIONS}

In this work, we have developed two models aiming to calculate the inductance reduction due to a whatsoever magnetization in a magnet; one model is based on energetic considerations, the other on the vector potential description. Energetic considerations are useful in problems where the magnetic field is known, whereas the vector potential is useful for numerical problems. Then, we treated the specific problem of the IFCCs in superconducting magnets, showing two ways for the calculation of the wire magnetization. We have shown two examples of comparison of the models with experimental data: an analytical example for a long quadrupole magnet and a numerical example for a short racetrack magnet; in both cases, the agreement between the models and the experimental data is very satisfying.

These models are a very important improvement for the quench protection study of superconducting accelerator magnets [5], providing more realistic values of the inductance of the magnets during fast discharges (with respect to the much too large values of static inductances) and better estimations of the maximum temperature during a quench and allowing the development of more performing magnets.

\section{ACKNOWLEDGMENTS}

The research leading to these results has received funding from the European Commission under the Transnational Access activity of the FP7 Research Infrastructures project EUCARD-2, Grant Agreement No. 312453, from the U.S. Department of Energy through the U.S. LHC Accelerator Research Program (LARP), and it is partly funded by the European Commission within the Framework Program 7 Capacities Specific Program, Grant Agreement No. 284404. The HiLumi LHC Design Study is included in the High Luminosity LHC project. 
[1] L. Rossi, Superconductivity: Its role, its success and its setbacks in the Large Hadron Collider of CERN, Supercond. Sci. Technol. 23, 10 (2010).

[2] G. Manfreda, G. Ambrosio, V. Marinozzi, T. Salmi, M. Sorbi, and G. Volpini, Quench protection study of the $\mathrm{Nb}_{3} \mathrm{Sn}$ low- $\beta$ quadrupole for the LHC luminosity upgrade, IEEE Trans. Appl. Supercond. 24, 4700405 (2013).

[3] L. Rossi, Fermilab Technical Division, Technical Report No. TD-12-11, 2012.

[4] V. Marinozzi, Master's thesis, University of Milan, 2013 (Fermilab Technical Division, Technical Report No. TD13-013, 2013).

[5] V. Marinozzi, G. Ambrosio, G. Bellomo, G. Chlachidze, H. Felice, M. Marchevsky, T. Salmi, M. Sorbi, and E. Todesco, Study of quench protection for the $\mathrm{Nb}_{3} \mathrm{Sn}$ low- $\beta$ quadrupole for the LHC luminosity upgrade(HiLumi-LHC), IEEE Trans. Appl. Supercond. 25, 4002905 (2015).

[6] M. N. Wilson, Superconducting Magnets (Clarendon, Oxford, 1983).

[7] M. Breschi, M. Massimini, P. L. Ribani, and V. Corato, Experimental and numerical analysis of interfilament resistances in $\mathrm{Nb}_{3} \mathrm{Sn}$ and $\mathrm{NbTi}$ strands, IEEE Trans. Appl. Supercond. 24, 1 (2014).

[8] E. Barzi, D. Turrioni, and A. V. Zlobin, Progress in $\mathrm{Nb}_{3} \mathrm{Sn}$ RRP strand studies and Rutherford cable development at FNAL, IEEE Trans. Appl. Supercond. 24, 1 (2014).

[9] I. Solomonovich Gradshteyn, A. Jeffrey, and I. Moiseevich Ryzhik, Table of integrals, series, and products, 4th corrected and revised ed., Chap. 2, p. 92 (Academic, New York, 1980). With corrigenda: loose-leaf ed. [in closed Ref.]: translated from the 4th Russian ed., Moscow, 1963.

[10] M. Sorbi, Lessons of Academic Course in Applied Superconductivity, Milan University (unpublished).

[11] L. Rossi and M. Sorbi, QLASA: A computer code for quench simulation in adiabatic multicoil superconducting windings, Nat. Inst. of Nucl. Phys. (INFN), Rome, Italy, Technical Report No. TC-04-13, 2004.

[12] H. Felice et al., Design of HQ - A high field large bore $\mathrm{Nb}_{3} \mathrm{Sn}$ quadrupole magnet for LARP, IEEE Trans. Appl. Supercond. 19, 1235 (2009).

[13] H. Bajas et al., in Proceedings of the Applied Superconductivity Conference, Portland, Oregon, USA, 2012 (unpublished).

[14] H. Bajas et al., Quench Analysis of High Current Density $\mathrm{Nb}_{3}$ Sn Conductors in Racetrack Coil ConFiguration, IEEE Trans. Appl. Supercond. (to be published).

[15] F. Bellina and E. Serra, Computation of the magnetostatic field by means of a mixed analytical-numerical procedure, IEEE Trans. Magn. 40, 834 (2004).

[16] J. W. Eaton, D. Bateman, and S. Hauberg, GNU Octave Vversion 3.0.1 Manual: A High-Level Interactive Language for Numerical Computations (CreateSpace Independent Publishing Platform, 2009), ISBN 1441413006.

[17] L.F. Shampine, Vectorized adaptive quadrature in MATLAB, J. Comput. Appl. Math. 211, 131 (2008). 